Journal of Engineering and Applied Sciences 15 (4): 898-907, 2020

ISSN: $1816-949 \mathrm{X}$

(C) Medwell Journals, 2020

\title{
Employing Symmetry Concept and Unsupervised Neural Network to Detect Abnormal Regions in IR Breast Thermograms
}

\author{
Rabab Saadoon Abdoon \\ Department of Physics, College of Science, University of Babylon, Hillah, Iraq
}

\begin{abstract}
Infrared thermography is one of many medical scanning for detecting breast cancer and other abnormalities. Women breasts possess a high degree of symmetry, this property is employed in this research to detect the presence of abnormalities in breast front view thermograms of left and right breasts by proposing symmetry line algorithm. Clustering process utilizing unsupervised Self Organization Feature Map (SOFM) neural network is a second proposed technique in this work to isolate and extract abnormal regions in IR breast thermograms. The results declared the efficiency of the proposed symmetry line algorithm, depending on the histogram and standard deviation calculated values to detect breast abnormalities in the experimental thermograms. As well as the results of the second proposed unsupervised neural network clustering method proved its effective and adequate performance, it succeeded to extract the cancer and other abnormal regions in the abnormal sets of breast thermograms.
\end{abstract}

Key words: Symmetry, unsupervised neural network, IR thermograms, breast cancer, infrared, thermography

\section{INTRODUCTION}

Imaging utilizing IR thermography was essentially, designed for detecting abnormal heat and any vascular development in the tissue of breast which are attributed to early growth of tumors. All tumors whether benign or malignant (cancer) need blood supply to nourish themselves for growth. The growth of these vascularities in abnormal patterns will create the heat and inflammation that can be detected by thermal imaging camera. Infrared thermography scanning is passive, non-invasive, painless and safe, since, it does not utilize any ionizing radiation (Umadevi et al., 2011).

Different image processing techniques were employed to segment IR thermography breast images to detect tumors and other abnormal regions. Some of these methods are contour based, region based and features based methods. Soft and hard clustering methods were utilized by many researchers. Other researchers proposed supervised neural network and many other methods, see for example Yang et al. (2007); Tang et al. (2008); Zadeh et al. (2011); Mohamed (2015); Al-Dergazly (2017); Abdoon (2018).

In this research, symmetry concept is employed to detect the presence of abnormal regions depending on the high degree of symmetry between the left and right breasts in the front view thermograms images by implementing symmetry line algorithm. As well as, unsupervised SOFM neural network is proposed here as clustering method to isolateand extract the breast abnormalities.
Symmetry line of breasts front view images: Women breasts possess a high degree of symmetry, even not perfectly symmetrical. The symmetry plane of the breasts front view image can be considered as a good approximation of the mid line halves by which two high level symmetrical left and right breasts can be separated. Line of symmetry algorithm was presented firstly by Abdoon et al. (2015) where they applied it on MRI brain images. This algorithm is applied in this work on IR thermal images of breasts due to the existence of high symmetry between the two breasts. More details for this algorithm can be found in Abdoon et al. (2015).

Symmetry concept is employed here to detect the abnormalities within two sets of IR breast front view thermograms by implementing line of symmetry algorithm. Mean and standard deviation values were calculated for the two patient's breasts (left and right breasts) images to investigate the presence of high differences between them after dividing breasts IR images into two halves. If high differences were found, each breast image is divided into five partitions in order to determine the position of the abnormal regions within the specified breast.

Unsupervised neural network: An unsupervised neural network called Self-Organization Feature Map (SOFM) is implemented in this work for segmenting process. This neural network was proposed by (Kohonen, 1989). SOFM which is a feed-forward neural network was frequently utilized to segment different types of medical images. It describes a mapping process from higher dimensions 
input space to another one of lower dimensions space. For more details about self-organization feature map neural network, see for instance ((Kohonen, 1995; Logeswari and Karnan, 2010; Mostafa and Soltanian-Zadeh, 2011).

In this research, SOFM is employed to cluster IR breast images in order to isolate and extract the abnormal regions in the tested thermograms depending on the image's histogram. The adopted SOFM network architecture has four major nodes in the first layer and three sub nodes in the hidden layer. The initial weights of this network were taken by investigating the image's histogram and selecting the most distinct peaks.

Experimental images: In this research, two sets of IR breast front view thermograms were utilized to examine the performance of the proposed techniques. First set consists of four images for normal cases whereas the second set consists of three images for abnormal cases. The experimental images were acquired from different internet website in 3 July and 5 October, 2018 from Tahoma Clinic Thermography; new mexic othermography.com; Natural Awakenings Magazine and health phreaks.com for the normal images, respectively. While the abnormal images are from: foundation for alternative and integrative medicine, image courtesy of Donna Tomay, breast thermography.com and thermography centers.com respectively. Figure 1 and 2 show these experimental images.

(a)

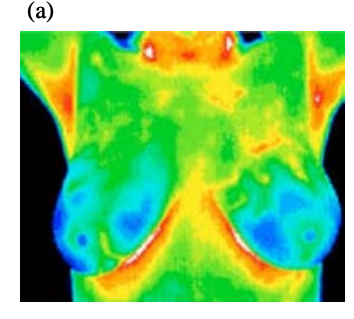

(b)

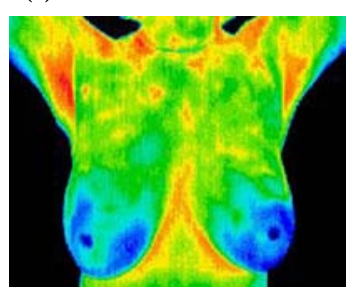

\section{MATERIALS AND METHODS}

Methodologies and experimental steps: In this section, the major work steps are presented. Firstly, the input colored thermograms tested images were converted to gray images to vacillate processing them. After that, the first proposed method, symmetry line algorithm will be implemented seeking for abnormality and then the second proposed unsupervised SOFM neural network clustering method will be implemented to isolate abnormalities in the abnormal tested images, as shown in the following:

Symmetry line: Symmetry concept is employed here to detect the abnormalities within the experimental IR thermograms of breast by implementing line of symmetry algorithm. This algorithm is applied in this study on IR thermal images of breasts due to the existence of high symmetry of the two breasts. Mean and standard deviation values were calculated for the two patient's breasts (left and right breasts) images to investigate abnormalities presence.

Unsupervised neural network clustering method: In this part of the work, unsupervised SOFMneural network clustering method is implemented to segment the abnormal thermograms and as a result extracting the abnormal regions. Figure 3 illustrates the steps of the proposed work.

(c)

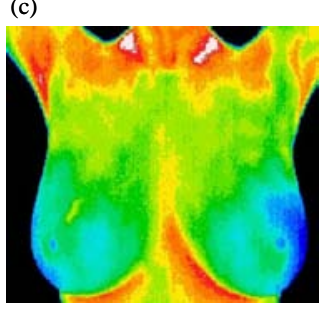

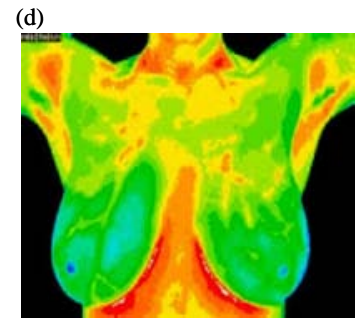

Fig. 1(a-d): First set of normal IR breast images

(a)

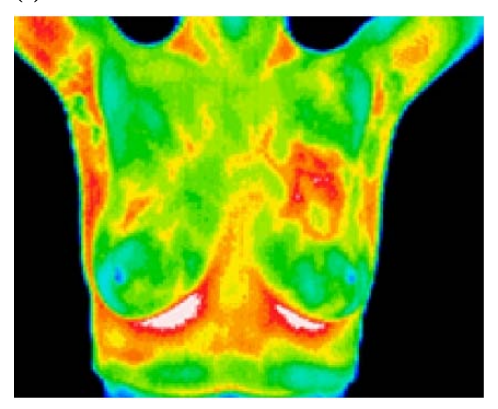

(b)

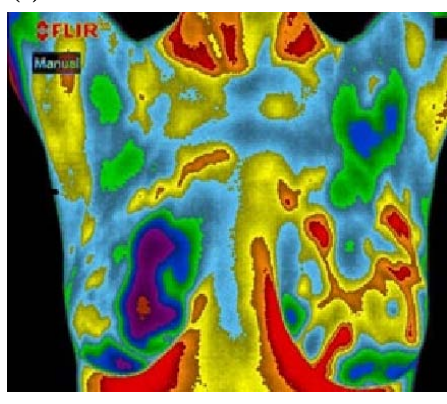

(c)

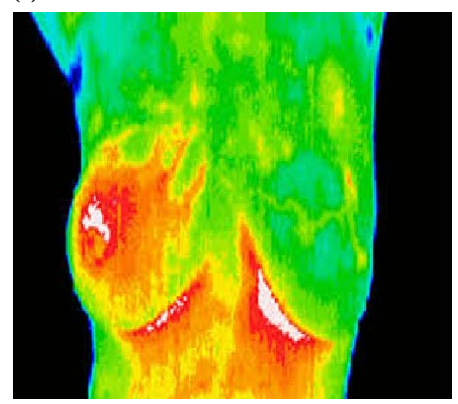

Fig. 2(a-c): Second set of abnormal IR breast images (a-b) Cancer cases and (c) Inflammatory carcinoma case 


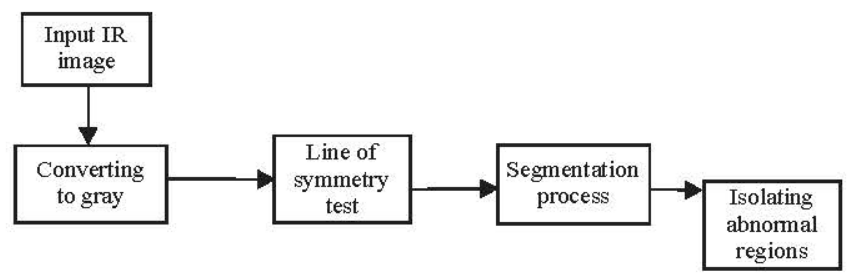

Fig. 3: Block diagram of the experimental steps

(a)
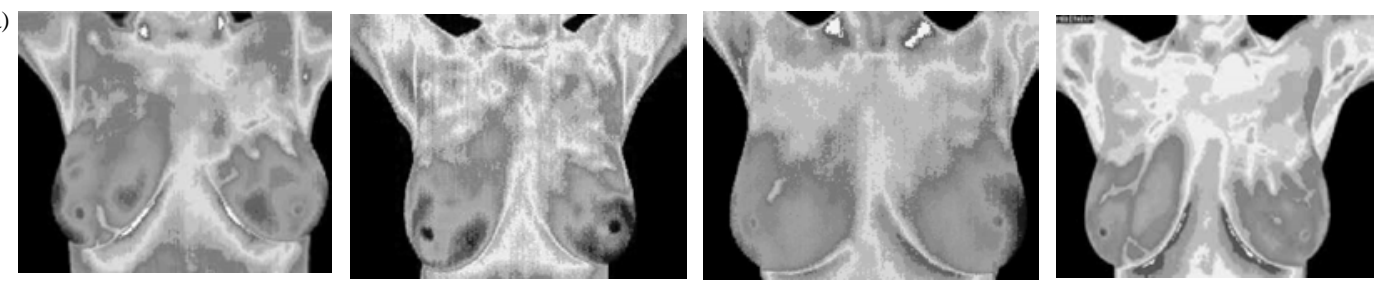

(b)
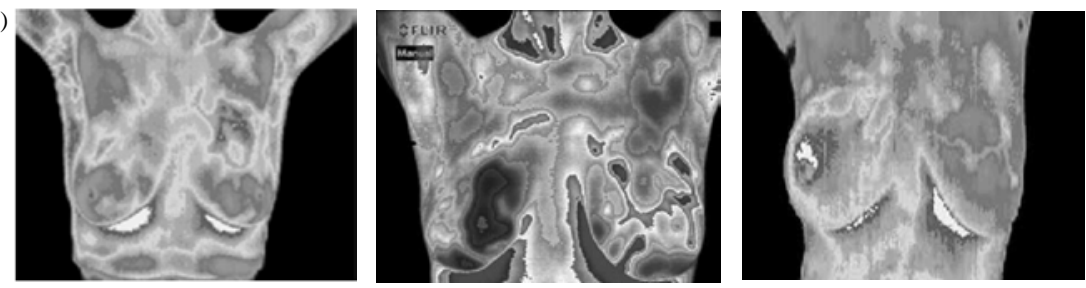

Fig. 4(a-b): Gray level images (a) First line for the normal set and (b) The second line for abnormal set

\begin{tabular}{|c|c|c|c|c|c|c|c|c|}
\hline \multirow[b]{2}{*}{ Images } & \multicolumn{2}{|l|}{ Image 1} & \multicolumn{2}{|l|}{ Image 2} & \multicolumn{2}{|l|}{ Image 3} & \multicolumn{2}{|l|}{ Image 4} \\
\hline & Left & Right & Left & Right & Left & Right & Left & Right \\
\hline$\overline{\text { Mean }}$ & 73.8934 & 65.7129 & 69.7103 & 57.1276 & 74.1033 & 64.0562 & 76.2124 & 68.0702 \\
\hline Standard deviation & 81.6588 & 82.9654 & 88.0659 & 84.0628 & 84.3401 & 81.0129 & 92.8670 & 90.5712 \\
\hline
\end{tabular}

\section{RESULTS AND DISCUSSION}

The first step in this research was converting the input IR thermograms into gray and the results of this step are presented in Fig. 4 for the two sets normal and abnormal images.

Line of symmetry: Symmetry line algorithm is implemented to investigate any asymmetry betweenthe (left and right breastswhich indicates the existence of abnormality regions in one half rather than the other. The results of implementing symmetry line algorithm on the first set of themograms: two halve's images their histograms and the histogram difference are showed in Fig. 5. Mean and standard deviation values of the two halve's images (left and right breasts) are calculated and presented in Table 1.

Figure 5 shows that the histograms of the two halves of each thermogram of the first set are very close to each other. Table 1 indicates that there is very small percent relative differences in the standard deviation values which are: $1.575,4.546,3.945$ and $2.472 \%$ for the four images, respectively.
Symmetry line algorithm is implemented on the second set of breast thermograms. From the results, it is figured out the existence of differences in halve's images histogram. As well as, there is a high percent relative differences of the standard deviation values between the left and right breast's images which are: 5.703, 8.849 and $24.026 \%$ for the three images, respectively, so, each thermogram's half is divided into five partitions to investigate the position of the abnormal regions. The results for abnormal set are presented in Fig. 6 and the calculated mean and standard deviation values of the two image's halves (left and right breasts) and their four partitions (neck and shoulders partition is excluded) are presented in Table 2 and 3.

Unsupervised SOFM neural network: Unsupervised SOFM clustering method is employed here to segment abnormal thermogram images in order to extract the abnormal regions depending on the image's histogram. After many experiments, it was found that the proper architecture of SOFM network has four major nodes in the first layer and three sub nodes in the hidden layer with 300 iterations. The initial weights of this network was 
J. Eng. Applied Sci., 15 (4): 898-907, 2020
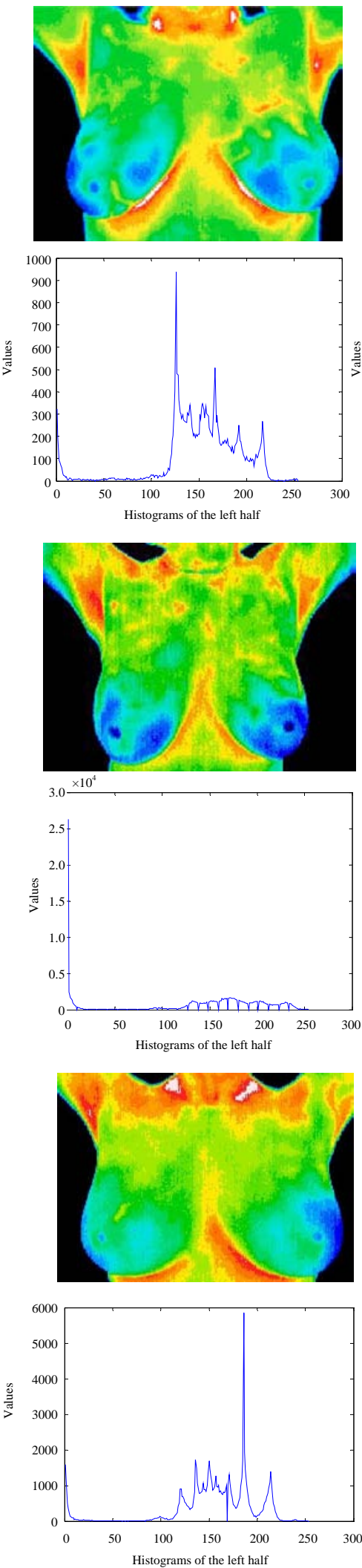
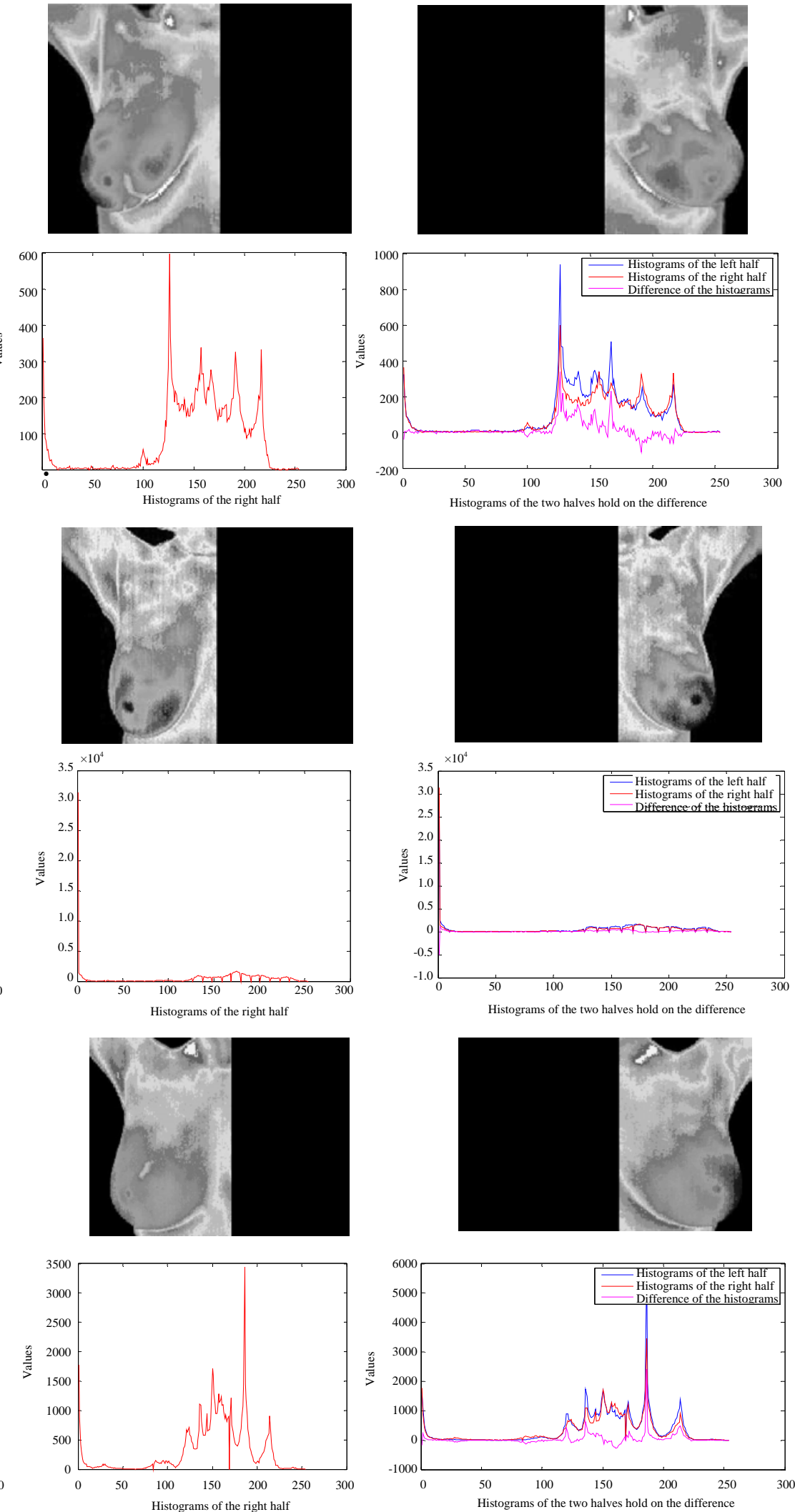

Fig. 5: Continue 

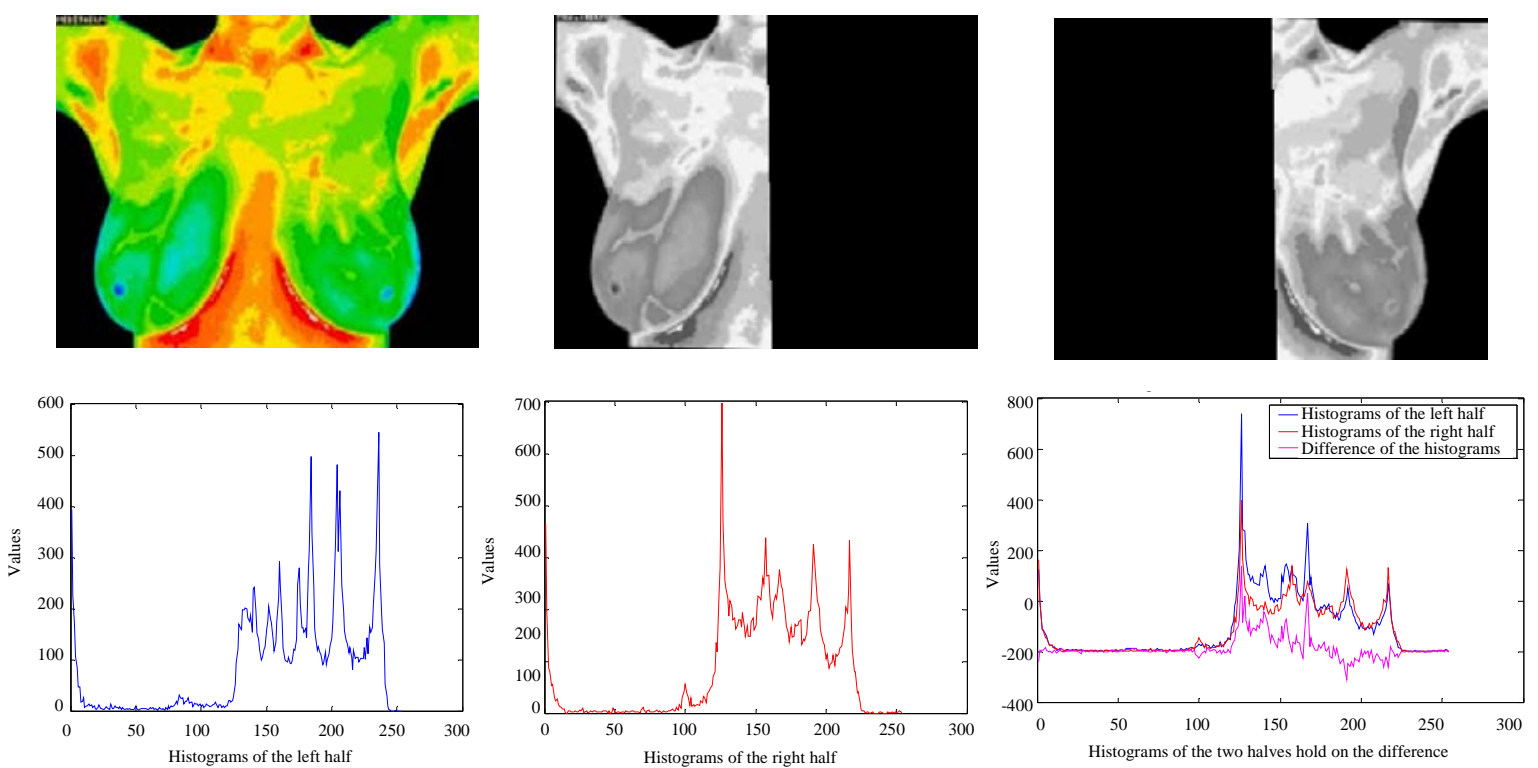

Fig. 5: Results of implementing symmetry line algorithm on normal set of IR thermograms. In first line of each group: the colored thermogram, left and right gray halves while in second line: histogram of left, right and the histogram differences hold on the two halve's histograms
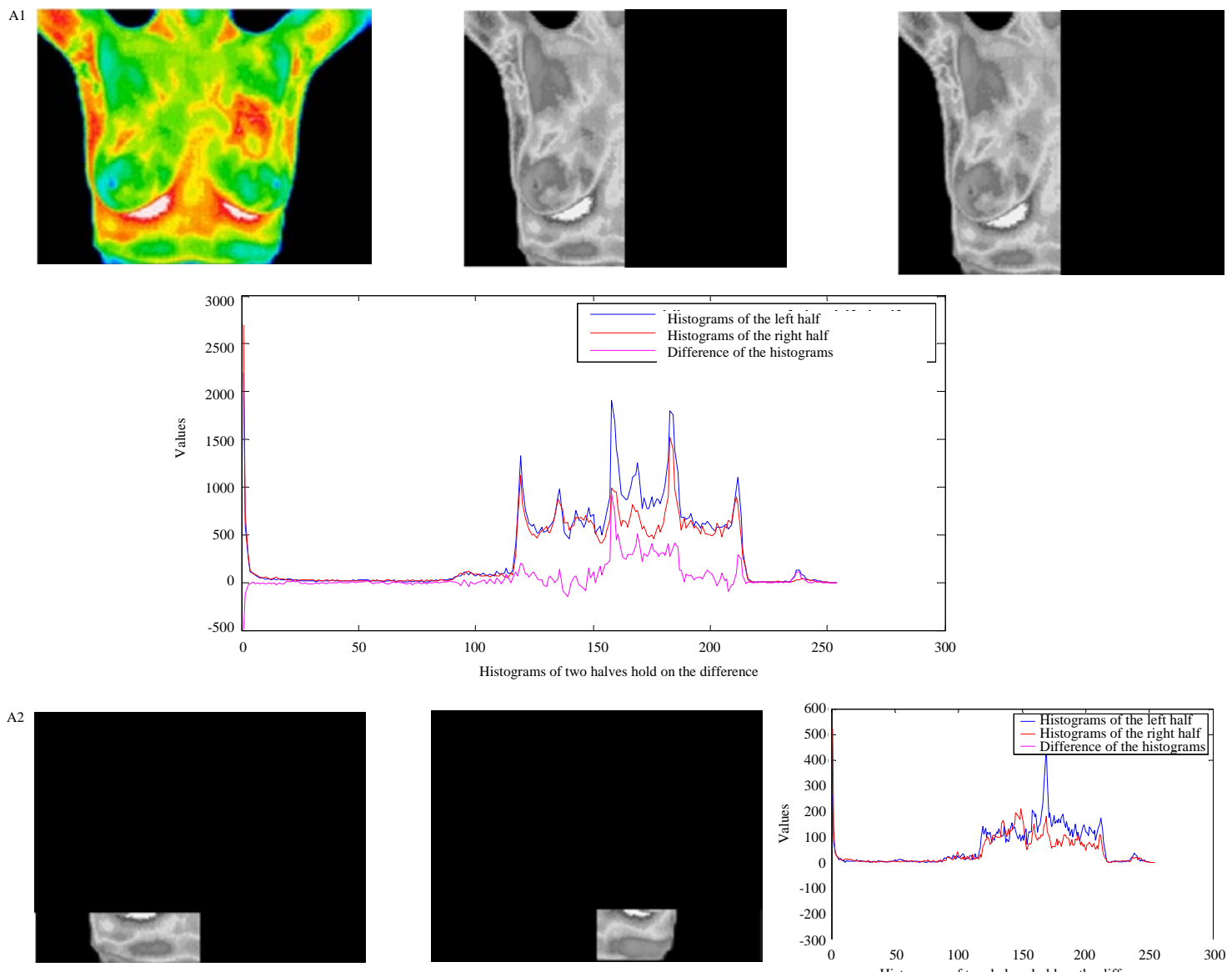

Fig. 6: Continue 

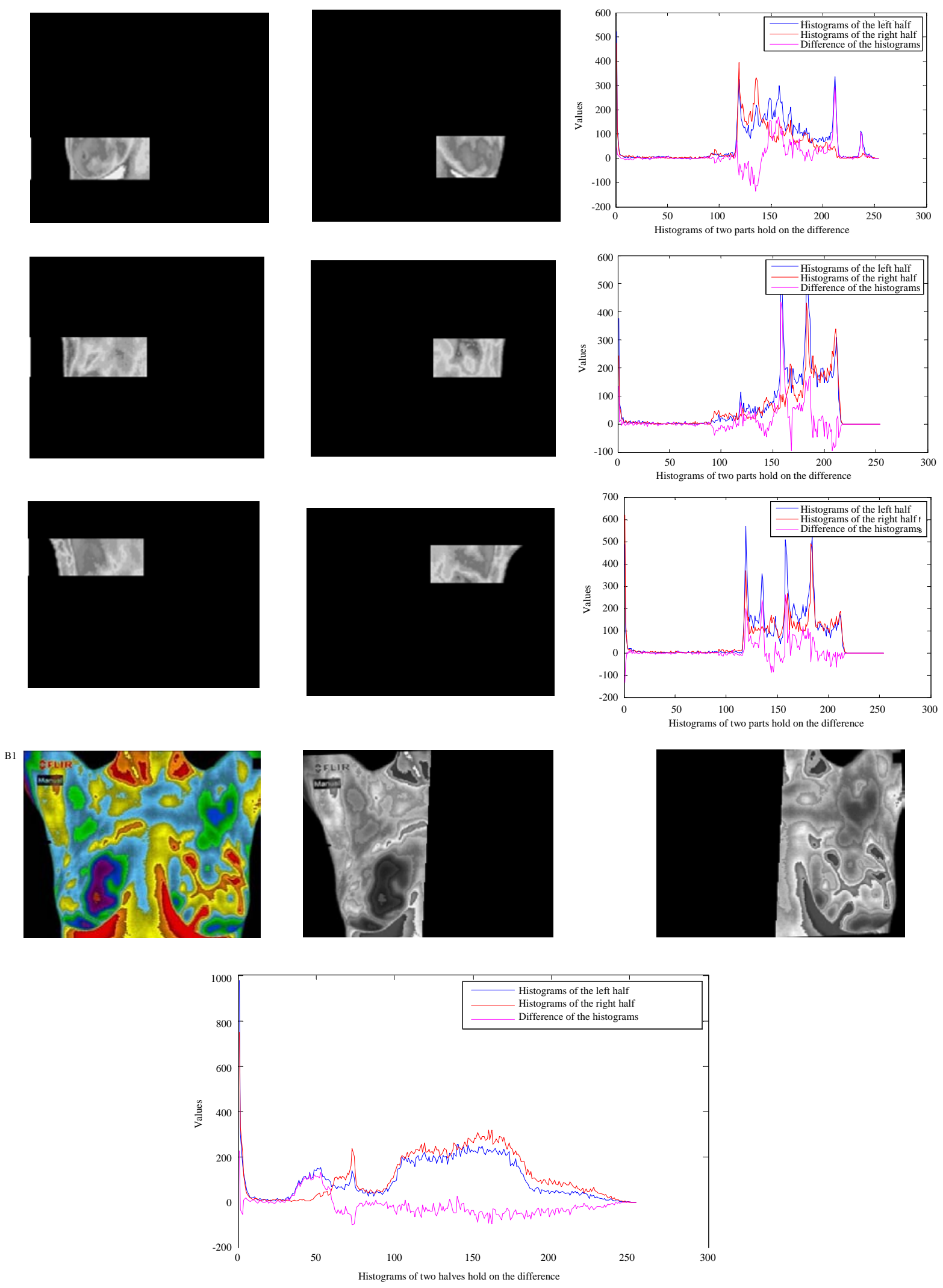

Fig. 6: Continue 

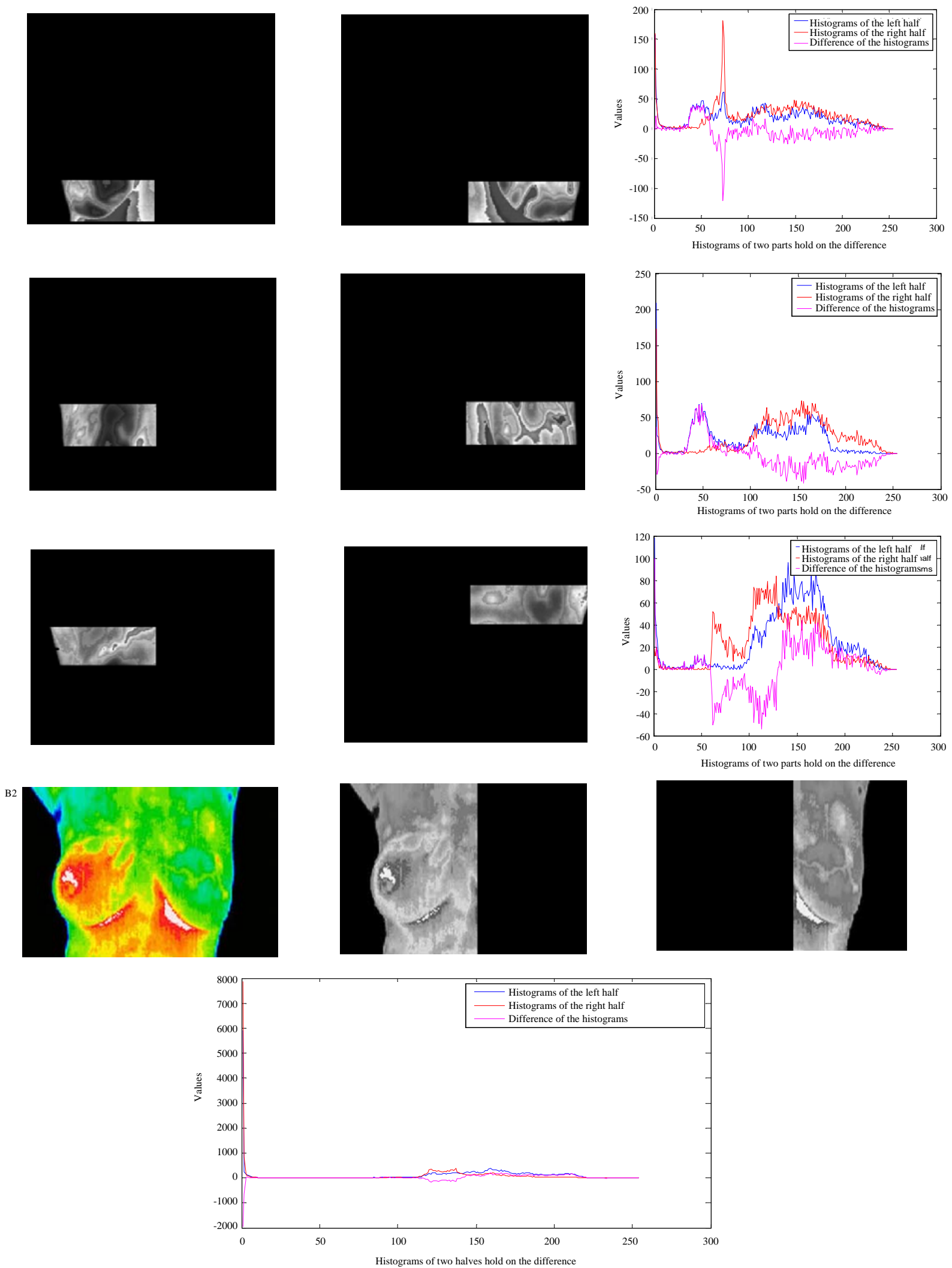

Fig. 6: Continue 
C2
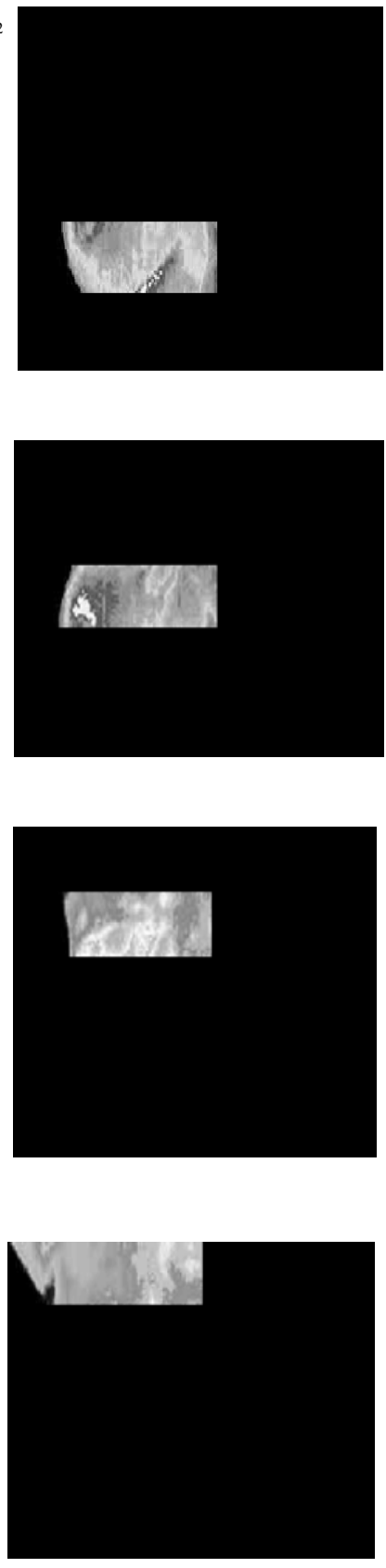
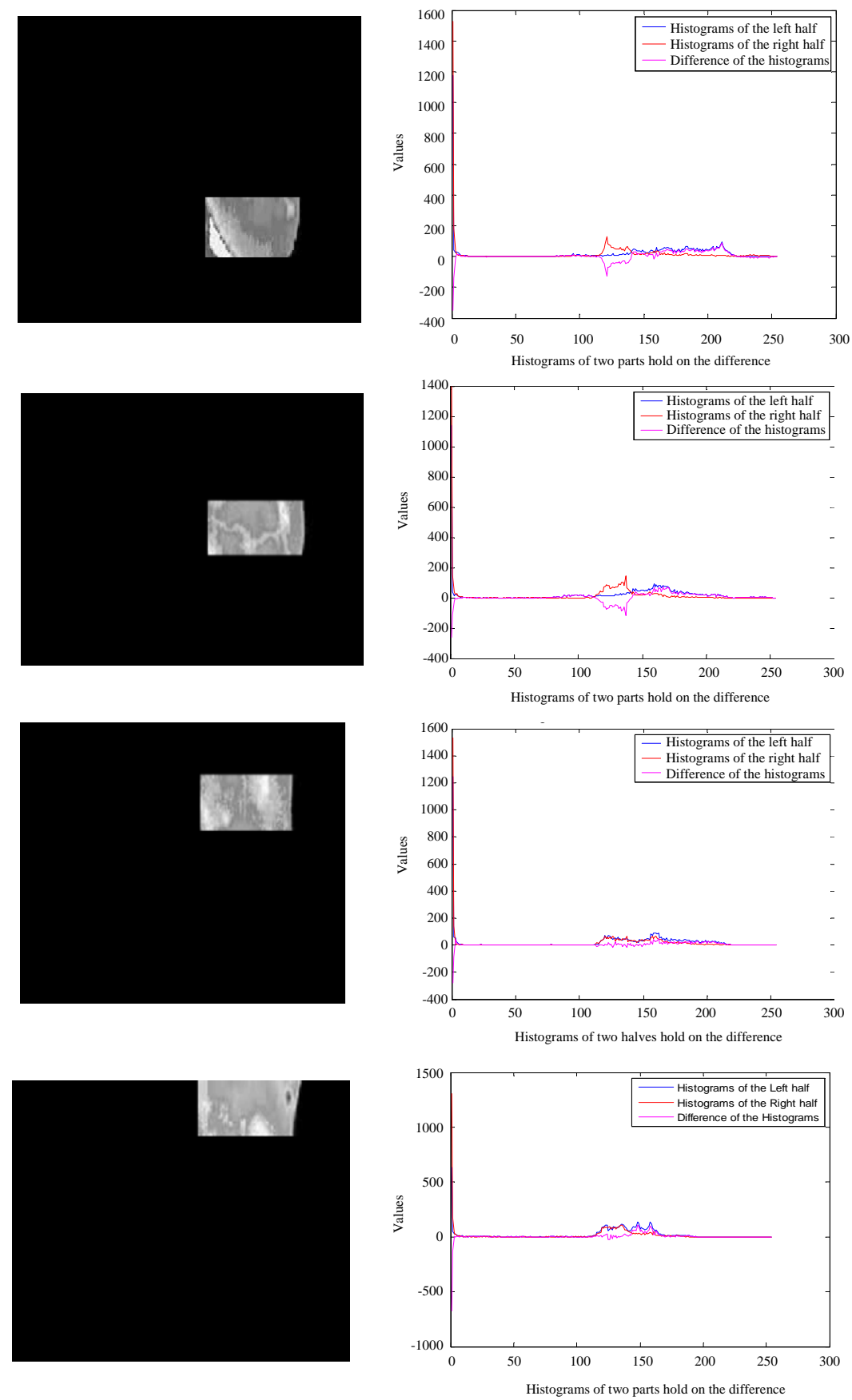

Fig. 6: Results of implementing symmetry line algorithm on abnormal set of IR thermograms. A1, B1 and $\mathrm{C} 1$ for the total left and right halve's images and difference histograms holds on the individual histograms of the three images respectively while $\mathrm{A} 2, \mathrm{~B} 2$ and $\mathrm{C} 2$ for the four partitions of left and right halves images and histograms of the three images, respectively

adopted depending on the most distinct peaks of the image's histogram. The results of this method are presented in Fig. 7.

Figure 7 illustrates the results of SOFM clustering method for the abnormal set of thermograms. First column shows the abnormal breast IR images, second column represents the black/white images of the isolated abnormal regions while the third column presents the colored extracted abnormal regions. Many morphological processes were needed to assist extracting the abnormal regions such as erosion, dilation and area selecting. It is cleared the effective performance of the proposed method in extracting the cancer and other abnormal regions. 

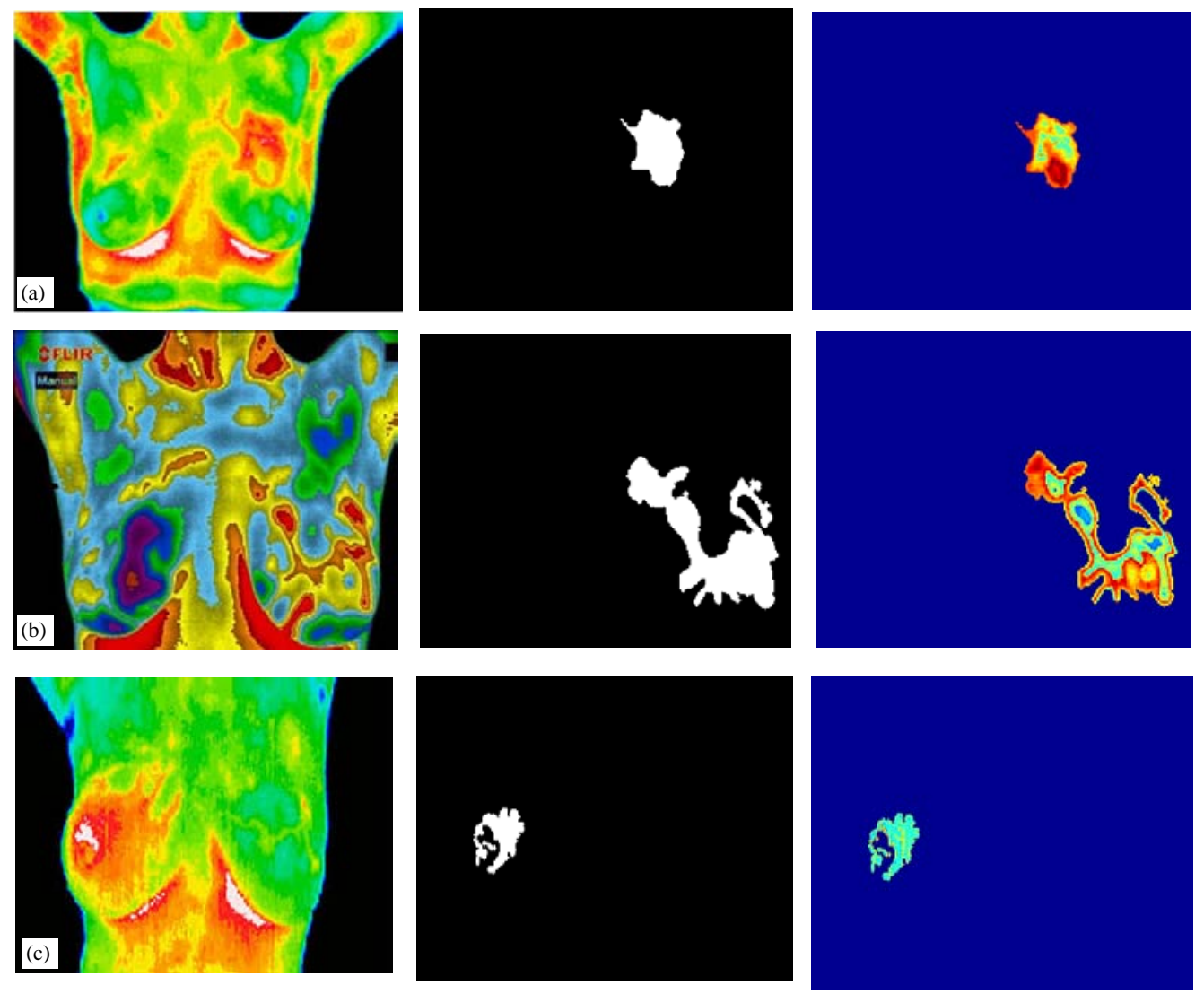

Fig. 7(a-c): Results of SOFM clustering method for the three abnormal IR breast images from first row to the last one, respectively

Table 2: Mean values of the left and right partitions halves of the abnormal set of images

\begin{tabular}{lllllll}
\hline Images & \multicolumn{2}{l}{ Mean calculated values } & & & & \\
\hline Image 1 & Left part & $62.1361^{*}$ & 10.8217 & 11.2340 & 12.5126 & 12.4087 \\
& Right part & $50.4544^{*}$ & 7.5071 & 8.0402 & 10.1966 & 10.6686 \\
Image 2 & Left part & $51.5251^{*}$ & 8.3859 & 8.5297 & 11.6377 & 14.4118 \\
& Right part & $65.2877^{*}$ & 11.1254 & 13.4571 & 13.3145 & 12.4353 \\
Image 3 & Left part & $66.2556^{*}$ & 12.7674 & 13.9728 & 12.9723 & 12.4621 \\
& Right part & $39.2009^{*}$ & 7.0286 & 7.8572 & 7.6826 & 8.1168 \\
\hline
\end{tabular}

*symbol for the total halves

Table 3: Standard deviation calculated values of the left and right partitions halves of the abnormal set images

\begin{tabular}{|c|c|c|c|c|c|c|}
\hline \multirow{2}{*}{$\begin{array}{l}\text { Image } \\
\text { Image } 1\end{array}$} & \multicolumn{6}{|c|}{ Standard deviation calculated values } \\
\hline & Left part & $82.8761^{*}$ & 41.8166 & 42.2157 & 45.6153 & 43.9193 \\
\hline & Right part & $78.4048^{*}$ & 34.6634 & 34.6801 & 41.9796 & 41.4006 \\
\hline Image 2 & Left part & $69.9869^{*}$ & 33.7185 & 32.7524 & 40.1275 & 45.7299 \\
\hline & Right part & $76.1800^{*}$ & 39.0300 & 44.6111 & 44.1149 & 40.3930 \\
\hline Image 3 & Left part & $81.4966^{*}$ & 46.0091 & 47.9927 & 44.4493 & 43.1947 \\
\hline & Right part & $65.7094^{*}$ & 32.9657 & 34.0551 & 31.8700 & 33.9993 \\
\hline
\end{tabular}

*symbol for the total right and left halves

\section{CONCLUSION}

Symmetry concept was employed in this study to investigate the presence of abnormal regions in IR breast front view thermograms by implementing symmetry line algorithm. The results declared the efficiency of this algorithm depending on the histogram and standard deviation calculated values to detect breast cancer and other abnormalities in the experimental thermograms. As well as, unsupervised SOFM neural network was utilized as clustering algorithm to isolate and extract abnormalities in the abnormal set of thermograms. The 
results of this technique proved that its effective and adequate performance, it succeeded to extract the abnormal regions in the abnormal tested thermograms.

\section{REFERENCES}

Abdoon, R.S., 2018. Utilizing image processing techniques for detecting breast abnormalities in thermography images. Int. J. Eng. Technol., 7: $2810-2817$.

Abdoon, R.S., L. Abood and S. Ali, 2015. Adaptive Techniques for Brain Tumor Detection in MRI. LAP LAMBERT Academic Publishing, Saarbrücken, Germany, ISBN-13:978-3659798634, Pages: 420.

Al-Dergazly, A.A., 2017. Thermal images segmentation for evaluation of breast cancer. Int. J. Sci. Res., 6: $230-242$.

Kohonen, T., 1989. Self-Organization and Associative Memory. 3rd Edn., Springer, New York, USA., ISBN: 9783540513872 , Pages: 312.

Kohonen, T., 1995. Self-Organizing Maps. 1st Edn., Springer, New York, USA.

Logeswari, T. and M. Karnan, 2010. An improved implementation of brain tumor detection using segmentation based on soft computing. J. Cancer Res. Exp. Oncol., 2: 006-014.

Mohamed, N.A., 2015. Breast cancer risk deteotion using digital infrared thermal images. Int. J. Bioinf. Biomed. Eng., 1: 185-194.
Mostafa J.M. and H. Soltanian-Zadeh, 2011. Medical Image Segmentation using Artificial Neural Networks. In: Artificial Neural NetworksMethodological Advances and Biomedical Applications, Suzuki, K. (Ed.). In-Tech Electronics Ltd., Hong Kong, ISBN: 978-953-307-243-2, pp: 121-138.

Tang, X., H. Ding, Y.E. Yuan and Q. Wang, 2008. Morphological measurement of localized temperature increase amplitudes in breast infrared thermograms and its clinical application. Biomed. Signal Process. Control, 3: 312-318.

Umadevi, V., S.V. Raghavan and S. Jaipurkar, 2011. Framework for estimating tumour parameters using thermal imaging. Indian J. Med. Res., 134: 725-731.

Yang, H.Q., S.S. Xie, Q.Y. Lin, Z. Ye, S.Q. Chen and H. Li, 2007. A new infrared thermal imaging and its preliminary investigation of Breast Disease Assessment. Proceedings of the 2007 IEEE/ICME International Conference on Complex Medical Engineering, May 23-27, 2007, IEEE, Beijing, China, pp: 1071-1074.

Zadeh, H.G., I.A. Kazerouni, J. Haddadnia, M. Rahmanian, R. Javidan and M.A. Dezfuli, 2011. Distinguish breast cancer based on thermal features in infrared images. Can. J. Image Process. Comput. Vision, 2: 54-58. 